\title{
Radial derivative on bounded symmetric domains
}

\author{
by \\ Guangbin Ren (Hefei) and Uwe KäHLer (Aveiro)
}

\begin{abstract}
We establish weighted Hardy-Littlewood inequalities for radial derivative and fractional radial derivatives on bounded symmetric domains.
\end{abstract}

1. Introduction. Let $\Omega$ be a bounded symmetric domain in the complex vector space $\mathbb{C}^{n}$. We always assume $0 \in \Omega$. Let $b$ be the Bergman-Shilov boundary of $\Omega$ with Lebesgue measure $\sigma$ such that $\sigma(b)=1$.

Let $k \in \mathbb{N} \cup\{0\}$ and define

$$
m_{k}=\left(\begin{array}{c}
n+k-1 \\
k
\end{array}\right) .
$$

In [4], Hua constructed a set $\left\{\varphi_{k v}: k \in \mathbb{N} \cup\{0\}, \nu=1, \ldots, m_{k}\right\}$ of homogeneous polynomials, which is complete and orthogonal on $\Omega$ and orthonormal on $b$. It is known that every holomorphic function $f$ in $\Omega$ has a series expansion (see [3]):

$$
f(z)=\sum_{k, v} a_{k, v} \varphi_{k, v}(z),
$$

where $\sum_{k, v}=\sum_{k=0}^{\infty} \sum_{\nu=1}^{m_{k}}$, and the convergence is uniform on compact subsets of $\Omega$. The coefficients are given by the formula

$$
a_{k, v}=\lim _{r \rightarrow 1} \int_{b} f(r \zeta) \overline{\varphi_{k, v}(\zeta)} d \sigma(\zeta) .
$$

In [6], Shi introduced the following fractional derivative $f^{[\beta]}$ and fractional integral $f_{[\beta]}$ :

$$
f^{[\beta]}(z)=\sum_{k, v} \frac{\Gamma(k+\beta+1)}{\Gamma(k+1)} a_{k, \nu} \varphi_{k, \nu}(z),
$$

2000 Mathematics Subject Classification: Primary 46E35; Secondary 47B38.

Key words and phrases: invariant harmonic functions, Bloch space.

Project supported by the NNSF of China (nos. 10001030, 19871081) and the Postdoctoral Fellowship of University of Aveiro, UID. 


$$
f_{[\beta]}(z)=\sum_{k, v} \frac{\Gamma(k+1)}{\Gamma(k+\beta+1)} a_{k, \nu} \varphi_{k, \nu}(z) .
$$

He studied the rate of growth of the integral means of holomorphic functions in terms of these operators. To state his result, we need some notation.

By $H(\Omega)$ we denote the holomorphic functions on $\Omega$. If $f \in H(\Omega)$, the integral mean $M_{q}(r, f), 0<q \leq \infty$, is defined by

$$
\begin{aligned}
M_{q}(r, f) & =\left\{\int_{b}|f(r \zeta)|^{q} d \sigma(\zeta)\right\}^{1 / q}, \quad 0<q<\infty ; \\
M_{\infty}(r, f) & =\sup \{|f(r \zeta)|: \zeta \in b\} .
\end{aligned}
$$

As usual, the symbol $A \simeq B$ means $C^{-1} B \leq A \leq C B$, where $C$ always denotes a positive constant, not necessarily the same at each occurrence and independent of $f$.

Theorem A $([6])$. Let $f \in H(\Omega), 0<p<\infty, 0<q \leq \infty, \alpha, \beta>0$. Then

$$
\int_{0}^{1}(1-r)^{p \alpha-1} M_{q}^{p}(r, f) d r \simeq \int_{0}^{1}(1-r)^{p(\alpha+\beta)-1} M_{q}^{p}\left(r, f^{[\beta]}\right) d r .
$$

Instead of fractional derivatives we shall consider the radial derivatives and study the rate of growth of integral means in weighted cases.

For $\beta>0$ and $s \geq 0$ we define the fractional radial derivative $R^{\beta, s}$ and the fractional radial integral $R_{\beta, s}$ :

$$
\begin{aligned}
& \left(R^{\beta, s} f\right)(z)=\sum_{k, v}(k+s)^{\beta} a_{k, \nu} \varphi_{k, \nu}(z), \\
& \left(R_{\beta, s} f\right)(z)=\sum_{\substack{k, v \\
k+s>0}}(k+s)^{-\beta} a_{k, \nu} \varphi_{k, \nu}(z) .
\end{aligned}
$$

It is clear that for any $f \in H(\Omega)$, we have $R^{\beta, s} f, R_{\beta, s} f \in H(\Omega)$ and

$$
\begin{aligned}
R^{\beta, s} R_{\beta, s} f & =R_{\beta, s} R^{\beta, s} f=f, & & s>0 ; \\
R^{\beta} R_{\beta} f & =R_{\beta} R^{\beta} f=f-f(0), & & s=0 .
\end{aligned}
$$

When $\beta=1$ and $s=0$ we denote by $R=R^{1,0}$ the radial derivative. The fractional radial derivative was considered by Burbea [2] in the unit ball.

To study the growth of integral means of a holomorphic function, we shall consider the following type of functions as weight functions, which was first introduced by Shields and Williams [7].

A positive continuous function $\varphi$ on $[0,1)$ is normal if there exist $0<$ $a<b, 0 \leq r_{0}<1$ such that 
(i) $\frac{\varphi(r)}{(1-r)^{a}}$ is nonincreasing in $\left[r_{0}, 1\right)$ and $\lim _{r \rightarrow 1} \frac{\varphi(r)}{(1-r)^{a}}=0$;

(ii) $\frac{\varphi(r)}{(1-r)^{b}}$ is nondecreasing in $\left[r_{0}, 1\right)$ and $\lim _{r \rightarrow 1} \frac{\varphi(r)}{(1-r)^{b}}=\infty$.

For example,

$$
\varphi(r)=(1-r)^{\alpha} \log ^{\beta} \frac{2}{1-r}, \quad \alpha>0, \beta \in \mathbb{R},
$$

are normal functions. We point out that normal functions of the form (1.2) appear naturally in the study of multipliers of Bloch space (see [9]). One can verify that $r_{0}$ is strictly positive for normal functions of the form (1.2) by applying the derivative test for monotonicity functions around $r=0$ and $r=1$.

Our main results are the following theorems.

Theorem 1.1. Let $f \in H(\Omega), \varphi$ be normal, $0<p, q \leq \infty, \beta>0$. Then

$$
\begin{aligned}
& \int_{0}^{1}(1-r)^{-1} \varphi^{p}(r) M_{q}^{p}(r, f) d r \\
& \simeq|f(0)|^{p}+\int_{0}^{1} r^{-p}(1-r)^{p \beta-1} \varphi^{p}(r) M_{q}^{p}\left(r, R^{\beta} f\right) d r .
\end{aligned}
$$

When $p=\infty$, the inequality is understood to be its limit case:

$$
M_{q}(r, f)=O\left(\varphi^{-1}(r)\right) \Leftrightarrow M_{q}\left(r, R^{\beta} f\right)=O\left((1-r)^{-\beta} \varphi^{-1}(r)\right)
$$

as $r \rightarrow 1^{-}$.

Notice that $R^{\beta} f(0)=0$ for any $f \in H(\Omega)$ and $\beta>0$. We shall show that the function $r^{-1} M_{q}\left(r, R^{\beta} f\right)$ is a nondecreasing continuous function of $r \in(0,1)$ (see Prop. 3.4). This implies that $r=0$ is not a singular point of the integral in (1.3).

Theorem 1.2. Let $f \in H(\Omega), \varphi$ be normal, $0<p, q \leq \infty, \beta>0, s>0$. Then

$$
\int_{0}^{1}(1-r)^{-1} \varphi^{p}(r) M_{q}^{p}(r, f) d r \simeq \int_{0}^{1}(1-r)^{p \beta-1} \varphi^{p}(r) M_{q}^{p}\left(r, R^{\beta, s} f\right) d r .
$$

When $p=\infty$, the inequality is understood to be its limit case:

$$
M_{q}(r, f)=O\left(\varphi^{-1}(r)\right) \Leftrightarrow M_{q}\left(r, R^{\beta, s} f\right)=O\left((1-r)^{-\beta} \varphi^{-1}(r)\right)
$$

as $r \rightarrow 1^{-}$.

2. Normal functions. In this section, we give some basic properties of normal functions. 
Lemma 2.1. Let $0 \leq t \leq r<1, s>0, \varphi$ be a normal function with constants $a, b, r_{0}$. Then $\varphi(r) \simeq \varphi\left(r^{s}\right)$ and

$$
\frac{\varphi(r)}{(1-r)^{a}} \leq C \frac{\varphi(t)}{(1-t)^{a}}, \quad \frac{\varphi(r)}{(1-r)^{b}} \geq C \frac{\varphi(t)}{(1-t)^{b}} .
$$

Notice that Lemma 2.1 says we may always assume $r_{0}=0$, since monotonicity holds in the whole interval $[0,1)$ up to a constant.

Proof of Lemma 2.1. We first prove that

$$
\frac{\varphi(r)}{(1-r)^{a}} \leq C \frac{\varphi(t)}{(1-t)^{a}}, \quad 0 \leq t \leq r<1 .
$$

Clearly, this is true when $r_{0} \leq t \leq r<1$ by the monotonicity of $\varphi(r) /(1-r)^{a}$ in $\left[r_{0}, 1\right)$.

Let $M, m$ be the maximum and minimum of $\varphi(r) /(1-r)^{a}$ in $\left[0, r_{0}\right]$, respectively. Since $\varphi$ is positive and continuous on $\left[0, r_{0}\right]$, we have $M \geq m$ $>0$. Again from the monotonicity of $\varphi(r) /(1-r)^{a}$ in $\left[r_{0}, 1\right)$,

$$
\frac{\varphi(r)}{(1-r)^{a}} \leq \frac{\varphi\left(r_{0}\right)}{\left(1-r_{0}\right)^{a}} \leq M, \quad r_{0} \leq r<1 .
$$

Hence $M$ is the maximum of $\varphi(r) /(1-r)^{a}$ in $[0,1)$. If $t \leq r_{0}$, then for any $0<r<1$,

$$
\frac{\varphi(r)}{(1-r)^{a}} \leq M \leq \frac{M}{m} \frac{\varphi(t)}{(1-t)^{a}} .
$$

The other part in (2.1) can be proved in a similar way.

To prove $\varphi(r) \simeq \varphi\left(r^{s}\right)$, we can assume $s \geq 1$ by symmetry. Then from (2.1),

$$
\varphi\left(r^{s}\right)=\frac{\varphi\left(r^{s}\right)}{\left(1-r^{s}\right)^{a}}\left(1-r^{s}\right)^{a} \geq C \frac{\varphi(r)}{(1-r)^{a}}(1-r)^{a}=C \varphi(r) .
$$

The reverse inequality is obtained if $a$ is replaced by $b$. This finishes the proof.

Lemma 2.2. Let $\delta>0, h:[0,1) \rightarrow[0, \infty)$ be measurable, and $\varphi$ be a normal function. If $1 \leq \lambda<\infty$, then

$$
\begin{aligned}
& \int_{0}^{1}(1-r)^{-1} \varphi^{\lambda}(r)\left\{\int_{0}^{r}(r-t)^{\delta-1} h(t) d t\right\}^{\lambda} d r \\
& \leq C \int_{0}^{1}(1-r)^{\lambda \delta-1} \varphi^{\lambda}(r) h^{\lambda}(r) d r
\end{aligned}
$$

The inequality remains true if $0<\lambda<1$ and $h$ is nondecreasing in $[0,1)$. 
Proof. When $1 \leq p<\infty$ and $\varphi(r)=(1-r)^{a}$ with $a>0$, the inequality is due to Hardy; namely

$$
\int_{0}^{1}(1-r)^{\lambda a-1}\left\{\int_{0}^{r}(r-t)^{\delta-1} h(t) d t\right\}^{\lambda} d r \leq C \int_{0}^{1}(1-r)^{\lambda(a+\delta)-1} h^{\lambda}(r) d r .
$$

Assume $1 \leq p<\infty$ and $\varphi$ is a normal function with constants $a, b, r_{0}$. Choose $\varepsilon>0$ so small that $\varepsilon / \lambda<a$. By Lemma 2.1, $\varphi(r) /(1-r)^{a}$ and $(1-r)^{a-\varepsilon / \lambda}$ are both nonincreasing in $[0,1)$ and so is their product $\varphi(r) /(1-r)^{\varepsilon / \lambda}$. More precisely,

$$
\frac{\varphi(r)}{(1-r)^{\varepsilon / \lambda}} \leq C \frac{\varphi(t)}{(1-t)^{\varepsilon / \lambda}}, \quad 0 \leq t \leq r<1 .
$$

Therefore,

$$
\begin{aligned}
& \int_{0}^{1}(1-r)^{-1} \varphi^{\lambda}(r)\left\{\int_{0}^{r}(r-t)^{\delta-1} h(t) d t\right\}^{\lambda} d r \\
& \leq C \int_{0}^{1}(1-r)^{\varepsilon-1}\left\{\int_{0}^{r}(r-t)^{\delta-1} \frac{\varphi(t)}{(1-t)^{\varepsilon / \lambda}} h(t) d t\right\}^{\lambda} d r .
\end{aligned}
$$

If we set $a=\varepsilon / \lambda$ and replace $h(t)$ by $\left(\varphi(t) /(1-t)^{\varepsilon / \lambda}\right) h(t)$ in $(2.3)$, then we know that the right hand side of the above inequality is less than

$$
C \int_{0}^{1}(1-r)^{\lambda \delta-1} \varphi^{\lambda}(r) h^{\lambda}(r) d r
$$

as desired. The inequality (2.2) can be written with $t$ replaced by $r t$ as follows:

$$
\begin{aligned}
\int_{0}^{1} r^{\lambda \delta}(1-r)^{-1} \varphi^{\lambda}(r)\left\{\int_{0}^{1}(1-t)^{\delta-1}\right. & h(r t) d t\}^{\lambda} d r \\
& \leq C \int_{0}^{1}(1-r)^{\lambda \delta-1} \varphi^{\lambda}(r) h^{\lambda}(r) d r
\end{aligned}
$$

Now let $0<\lambda<1$ and $h$ be nonincreasing in $[0,1)$. To prove that $(2.2)$ or, equivalently, (2.4) still holds in this case, it is sufficient to show that

$$
\left\{\int_{0}^{1}(1-t)^{\delta-1} h(r t) d t\right\}^{\lambda} \leq C(\lambda, \delta) \int_{0}^{1}(1-t)^{\lambda \delta-1} h^{\lambda}(r t) d t
$$

which can be proved by using the following partition of $[0,1)$ :

$$
0=t_{0}<t_{1}<\ldots<1,
$$


where $t_{j}=1-1 / 2^{j}$. Indeed,

$$
\begin{aligned}
& \left(\int_{0}^{1}(1-t)^{\delta-1} h(r t) d t\right)^{\lambda}=\left(\sum_{j=1}^{\infty} \int_{t_{j-1}}^{t_{j}}(1-t)^{\delta-1} h(r t) d t\right)^{\lambda} \\
& \quad \leq \sum_{j=1}^{\infty}\left(\int_{t_{j-1}}^{t_{j}}(1-t)^{\delta-1} d t\right)^{\lambda} \sup _{t \in\left[t_{j-1}, t_{j}\right)} h^{\lambda}(r t) \leq C \sum_{j=1}^{\infty} 2^{-\lambda \delta} \sup _{t \in\left[t_{j-1}, t_{j}\right)} h^{\lambda}(r t) \\
& \quad \leq C \sum_{j=1}^{\infty} \int_{t_{j}}^{t_{j+1}}(1-t)^{\lambda \delta-1} h^{\lambda}(r t) d t \leq C \int_{0}^{1}(1-t)^{\lambda \delta-1} h^{\lambda}(r t) d t .
\end{aligned}
$$

Lemma 2.3. Let $0<\lambda<\infty, \delta>0, \mu>0, h:[0,1) \rightarrow[0, \infty)$ be nondecreasing, and $\varphi$ be a normal function. Then

$$
\begin{aligned}
\int_{0}^{1} r^{\lambda \delta}(1-r)^{-1} \varphi^{\lambda}(r)\left\{\int_{0}^{1}\left(\log \frac{1}{t}\right)^{\delta-1} t^{\mu-1}\right. & h(r t) d t\}^{\lambda} d r \\
& \lesssim \int_{0}^{1}(1-r)^{\lambda \delta-1} \varphi^{\lambda}(r) h^{\lambda}(r) d r .
\end{aligned}
$$

Proof. Let $s=1+1 / \mu$ and set $r=u^{s}, t=v^{s}$. We have

$$
\begin{aligned}
\int_{0}^{1} r^{\lambda \delta}(1-r)^{-1} \varphi^{\lambda}(r)\left\{\int_{0}^{1}\left(\log \frac{1}{t}\right)^{\delta-1} t^{\mu-1} h(r t) d t\right\}^{\lambda} d r \\
\quad \leq C \int_{0}^{1} u^{s \lambda \delta+s-1}(1-u)^{-1} \varphi^{\lambda}(u)\left\{\int_{0}^{1}\left(\log \frac{1}{v}\right)^{\delta-1} v^{s \mu-1} h\left(u^{s} v^{s}\right) d v\right\}^{\lambda} d u \\
\leq C \int_{0}^{1} u^{\lambda \delta}(1-u)^{-1} \varphi^{\lambda}(u)\left\{\int_{0}^{1}(1-v)^{\delta-1} h\left(u^{s} v^{s}\right) d v\right\}^{\lambda} d u .
\end{aligned}
$$

The last step came from the simple inequality

$$
v^{\sigma}\left(\log \frac{1}{v}\right)^{\delta-1} \leq C(1-v)^{\delta-1}, \quad v \in(0,1)
$$

for any $\sigma>0$, since it holds as $v \rightarrow 0^{+}$and $v \rightarrow 1^{-}$.

Combining the above result with (2.4) we obtain

$$
\begin{aligned}
\int_{0}^{1} r^{\lambda \delta}(1-r)^{-1} \varphi^{\lambda}(r)\left\{\int_{0}^{1}\left(\log \frac{1}{t}\right)^{\delta-1}\right. & \left.t^{\mu-1} h(r t) d t\right\}^{\lambda} d r \\
& \lesssim \int_{0}^{1}(1-r)^{\lambda \delta-1} \varphi^{\lambda}(r) h^{\lambda}\left(r^{1+1 / \mu}\right) d r .
\end{aligned}
$$

The assertion now follows from the fact that $h$ is nondecreasing. 
3. Radial derivative. Before the proof of the main results, we need some preparations.

Proposition 3.1. Suppose $f \in H(\Omega), \beta>0,0<q \leq \infty, s \geq 0$, $0<r<1$, and set $u=\min (1, q)$. Then there exists a constant $C=C(q, \beta, n)$ such that

$$
M_{q}^{u}\left(r, R_{\beta, s} f\right) \leq C \int_{0}^{1}\left(\log \frac{1}{\varrho}\right)^{\beta u-1} \varrho^{u s-1} M_{q}^{u}(r \varrho, f) d \varrho .
$$

Proof. First we give an integral formula for the radial integral operator. We always assume that $s>0$ or $s=0$ and $f(0)=0$. Then for any $f \in H(\Omega)$ we have

$$
R_{\beta, s} f(z)=\frac{1}{\Gamma(\beta)} \int_{0}^{1}\left(\log \frac{1}{\varrho}\right)^{\beta-1} \varrho^{s-1} f(\varrho z) d \varrho .
$$

To prove this, let $f(z)=\sum_{k, v} a_{k, v} \varphi_{k, v}(z)$; then

$$
\begin{aligned}
\frac{1}{\Gamma(\beta)} \int_{0}^{1}\left(\log \frac{1}{\varrho}\right)^{\beta-1} & \varrho^{s-1} f(\varrho z) d \varrho \\
& =\sum_{k, v} a_{k, v} \varphi_{k, v}(z) \frac{1}{\Gamma(\beta)} \int_{0}^{1}\left(\log \frac{1}{\varrho}\right)^{\beta-1} \varrho^{k+s-1} d \varrho .
\end{aligned}
$$

The formula (3.2) now follows from the identity

$$
\frac{1}{\Gamma(\beta)} \int_{0}^{1}\left(\log \frac{1}{\varrho}\right)^{\beta-1} \varrho^{k+s-1} d \varrho=\frac{1}{\Gamma(\beta)} \int_{0}^{\infty} t^{\beta-1} e^{-(k+s) t} d t=(k+s)^{-\beta}
$$

for any $k+s>0$ with $k \in \mathbb{N} \cup\{0\}$.

If $1 \leq q \leq \infty$, the inequality (3.1) is a direct corollary of (3.2) and Minkowski's inequality. Now assume $0<q<1$. We introduce a new partition of $(0,1)$ by setting

$$
0<\ldots<\varrho_{-2}<\varrho_{-1}<\varrho_{0}<\varrho_{1}<\varrho_{2}<\ldots<1
$$

with

$$
\varrho_{0}=\frac{1}{2}, \quad \varrho_{j}=\varrho_{j-1}^{1 / 2} \quad(j \in \mathbb{Z}) .
$$

By a simple computation for any $\beta>0$,

$$
\int_{\varrho_{j-1}}^{\varrho_{j}}\left(\log \frac{1}{\varrho}\right)^{\beta-1} \varrho^{-1} d \varrho=-\left.\frac{1}{\beta}\left(\log \frac{1}{\varrho}\right)^{\beta}\right|_{\varrho_{j-1}} ^{\varrho_{j}}=\frac{2^{\beta}-1}{\beta}\left(\log \frac{1}{\varrho_{j}}\right)^{\beta} .
$$


Assume $s \geq 0$. Then $\varrho^{s} \leq \varrho_{j}^{s}$ for $\varrho \in\left[\varrho_{j-1}, \varrho_{j}\right]$, and $\varrho_{j}^{q s} \leq \varrho^{q s}$ for $\varrho \in$ $\left[\varrho_{j}, \varrho_{j+1}\right]$. Therefore,

$$
\begin{aligned}
\left(\int_{\varrho_{j-1}}^{\varrho_{j}}\left(\log \frac{1}{\varrho}\right)^{\beta-1} \varrho^{s-1} d \varrho\right)^{q} & \leq C \varrho_{j}^{q s}\left(\log \frac{1}{\varrho_{j}}\right)^{q \beta} \\
& \leq C \int_{\varrho_{j}}^{\varrho_{j}+1}\left(\log \frac{1}{\varrho}\right)^{q \beta-1} \varrho^{q s-1} d \varrho .
\end{aligned}
$$

Combining this with (3.2), we have

$$
\begin{aligned}
\left|R_{\beta, s} f(r \zeta)\right|^{q} & \leq C\left\{\int_{0}^{1}\left(\log \frac{1}{\varrho}\right)^{\beta-1} \varrho^{s-1}|f(r \varrho \zeta)| d \varrho\right\}^{q} \\
& =C\left\{\sum_{j=-\infty}^{\infty} \int_{\varrho_{j-1}}^{\varrho_{j}}\left(\log \frac{1}{\varrho}\right)^{\beta-1} \varrho^{s-1}|f(r \varrho \zeta)| d \varrho\right\}^{q} \\
& \leq C \sum_{j=-\infty}^{\infty}\left(\int_{\varrho_{j-1}}^{\varrho_{j}}\left(\log \frac{1}{\varrho}\right)^{\beta-1} \varrho^{s-1} d \varrho\right)^{q} \sup _{\varrho \in\left(\varrho_{j-1}, \varrho_{j}\right)}|f(r \varrho \zeta)|^{q} \\
& \leq C \sum_{j=-\infty}^{\infty} \int_{\varrho_{j}}^{\varrho_{j+1}}\left(\log \frac{1}{\varrho}\right)^{q \beta-1} \varrho_{\varrho^{q s-1} d \varrho \sup _{\varrho \in\left(\varrho_{j-1}, \varrho_{j}\right)}|f(r \varrho \zeta)|^{q} .}
\end{aligned}
$$

Since Theorem 3 of [1] shows

$$
\int_{b} \sup _{\varrho \in\left(\varrho_{j-1}, \varrho_{j}\right)}|f(r \zeta)|^{q} d \sigma(\zeta) \leq C \sup _{\varrho \in\left(\varrho_{j-1}, \varrho_{j}\right)} \int_{b}|f(r \zeta)|^{q} d \sigma(\zeta),
$$

it follows that

$$
M_{q}^{q}\left(r, R_{\beta, s} f\right) \leq C \sum_{j=-\infty}^{\infty} \int_{\varrho_{j}}^{\varrho_{j+1}}\left(\log \frac{1}{\varrho}\right)^{q \beta-1} \varrho^{q s-1} d \varrho \sup _{\varrho \in\left(\varrho_{j-1}, \varrho_{j}\right)} M_{q}^{q}(r \varrho, f) .
$$

Now, from the monotonicity of integral means, we have

$$
\sup _{\varrho \in\left(\varrho_{j-1}, \varrho_{j}\right)} M_{q}^{q}(r \varrho, f) \leq \inf _{\varrho \in\left(\varrho_{j}, \varrho_{j+1}\right)} M_{q}^{q}(r \varrho, f),
$$

so that

$$
\begin{aligned}
M_{q}^{q}\left(r, R_{\beta, s} f\right) & \leq C \sum_{j=-\infty}^{\infty} \int_{\varrho_{j}}^{\varrho_{j+1}}\left(\log \frac{1}{\varrho}\right)^{q \beta-1} \varrho^{q s-1} M_{q}^{q}(r \varrho, f) d \varrho \\
& \leq C \int_{0}^{1}\left(\log \frac{1}{\varrho}\right)^{q \beta-1} \varrho^{q s-1} M_{q}^{q}(r \varrho, f) d \varrho .
\end{aligned}
$$

This completes the proof of Proposition 3.1. 
Proposition 3.2. If $f \in H(\Omega), \beta>0, s \geq 0$, and $0<q \leq \infty$, then for any $0 \leq r<1$,

$$
M_{q}\left(r, R^{\beta, s} f\right) \leq C(1-r)^{-\beta} M_{q}(r, f) .
$$

Proof. We first treat the special case $\Omega=U$, the unit disc in $\mathbb{C}$.

For $0<\eta<1$, let $S_{\eta}(\theta)$ be the Stoltz approximation domain. More precisely, it is an open subset of $U$ bounded by the two tangents from the point $e^{i \theta}$ to the circle with center 0 and radius $\eta$, together with the longer arc of this circle between the points of contact. Let $\beta>0, s \geq 0$, and $0 \leq \varrho<1$. We claim that

$$
\left|R^{\beta, s} f\left(\varrho e^{i \theta}\right)\right| \leq C(1-\varrho)^{-\beta} \sup _{z \in S_{\eta}(\theta)}|f(z)|, \quad \forall f \in H(U) .
$$

Assume $\beta=m$ is a positive integer and let $f \in H(U)$. It follows from

$$
R^{m, s} f(z)=\left(z \frac{d}{d z}+s\right)^{m} f(z)
$$

and the Cauchy integral formula that

$$
R^{m, s} f(z)=\frac{1}{2 \pi i} \int_{\Gamma} \frac{P(\zeta, z) f(\zeta)}{(\zeta-z)^{m+1}} d \zeta,
$$

where $P(\zeta, z)$ is a polynomial of $\zeta$ and $z$, and

$$
\Gamma=\Gamma(z):=\{\zeta \in \mathbb{C}:|\zeta-z|=\eta(1-|z|) / 2\} .
$$

Let $z=\varrho e^{i \theta}$. Since $\Gamma \subset S_{\eta}(\theta)$, we have

$$
\left|R^{m, s} f\left(\varrho e^{i \theta}\right)\right| \leq C(1-\varrho)^{-m} \sup _{z \in S_{\eta}(\theta)}|f(z)| .
$$

Now suppose $\beta$ is a noninteger and $s>0$. Pick the positive integer $m$ such that $\beta<m<\beta+1$. Since

$$
R^{\beta, s} f=R_{m-\beta, s} R^{m, s} f
$$

and $\log (1 / x) \geq 1-x$ for $x>0$, it can be deduced from (3.2) and (3.4) that

$$
\begin{aligned}
\left|R^{\beta, s} f\left(\varrho e^{i \theta}\right)\right| & \leq C \int_{0}^{1}\left(\log \frac{1}{r}\right)^{m-\beta-1} r^{s-1}\left|R^{m, s} f\left(r \varrho e^{i \theta}\right)\right| d r \\
& \leq C \int_{0}^{1} \frac{(1-r)^{m-\beta-1} r^{s-1}}{(1-r \varrho)^{m}} d r \sup _{z \in S_{\eta}(\theta)}|f(z)| \\
& \leq C(1-\varrho)^{\beta} \sup _{z \in S_{\eta}(\theta)}|f(z)| .
\end{aligned}
$$

This proves the claim.

Fix $0<r<1$, let $g=f_{r}=f(r x)$ and $g_{\zeta}(\lambda)=g(\lambda \zeta)$ for $\lambda \in \mathbb{C}$ and $\zeta \in b$, the Bergman-Shilov boundary. Then $g_{\zeta} \in H(U)$. By the claim and 
Hardy-Littlewood maximum theorem, we have

$$
M_{q}^{q}\left(r, R^{\beta, s} g_{\zeta}\right) \leq C(1-r)^{-\beta q}\left\|g_{\zeta}\right\|_{q}^{q} .
$$

Integrating both sides over $b$, from the formulas $\left(R^{\beta, s} g_{\zeta}\right)(\lambda)=\left(R^{\beta, s}\right) g(\lambda \zeta)$ and

$$
\frac{1}{2 \pi} \int_{b} d \sigma(\zeta) \int_{0}^{2 \pi} h\left(e^{i \theta} \zeta\right) d \theta=\int_{b} h(\zeta) d \sigma(\zeta), \quad h \in L^{1}(\sigma),
$$

we get the desired inequalities.

Remark 3.3. Note that if $s=0$, the polynomial $P(\zeta, z)$ in (3.4) has a factor $z$, so that the result can be strengthened for $s=0$ :

$$
M_{q}\left(r, R^{\beta, 0} f\right) \leq C r(1-r)^{-\beta} M_{q}(r, f) .
$$

Proposition 3.4. Let $\Omega$ be a bounded symmetric domain and $0<q$ $\leq \infty$. If $f \in H(\Omega)$ with $f(0)=0$, then $r^{-1} M_{q}(r, f)$ is a nondecreasing continuous function of $r \in(0,1)$.

Proof. There are two cases to consider.

CASE (i): $\Omega=U$. Since $f$ is holomorphic in $U$ and $f(0)=0$, we can take $h \in H(U)$ such that

$$
f(z)=z h(z), \quad z \in U .
$$

Thus $r^{-1} M_{q}(r, f)=M_{q}(r, h)$, which is a nondecreasing continuous function of $r \in(0,1)$.

CASE (ii): general case. Fix $0<r<1$, and let $f_{\zeta}(\lambda)=f(\lambda \zeta)$ for $\lambda \in \mathbb{C}$ and $\zeta \in b$. Then $f_{\zeta} \in H(U)$. From case (i), we know that $r^{-1} M_{q}\left(r, f_{\zeta}\right)$ is a nondecreasing continuous function of $r \in(0,1)$. Namely, for $0<r<\varrho$,

$$
r^{-1}\left\{\frac{1}{2 \pi} \int_{0}^{2 \pi}\left|f\left(r e^{i \theta} \zeta\right)\right|^{q} d \theta\right\}^{1 / q} \leq \varrho^{-1}\left\{\frac{1}{2 \pi} \int_{0}^{2 \pi}\left|f\left(\varrho e^{i \theta} \zeta\right)\right|^{q} d \theta\right\}^{1 / q} .
$$

On taking $q$ th powers and applying $\int_{\Omega} \cdot d \sigma(\zeta)$ to both sides, from (3.5) we deduce that $r^{-q} M_{q}^{q}(r, f) \leq \varrho^{-q} M_{q}^{q}(\varrho, f)$, so that

$$
r^{-1} M_{q}(r, f) \leq \varrho^{-1} M_{q}(\varrho, f)
$$

for any $0<r<\varrho$ and $0<q<\infty$. By the limit process, this also holds for $q=\infty$.

Now, we come to the proof of the main results.

Proof of Theorem 1.1. The inequality " $\gtrsim$ " in Theorem 1.1 is a direct corollary of Remark 3.3, the fact that $f(0)=M_{q}(0, f)$, and the monotonicity of $M_{q}(r, f)$. 
To prove the converse, we first consider the case of $0<p<\infty$. Define $u=\min \{q, 1\}$. Let $r=s^{p \beta+1}$. Then for any $f \in H(\Omega)$ we have

$$
\int_{0}^{1}(1-r)^{-1} \varphi^{p}(r) M_{q}^{p}\left(r, R_{\beta} f\right) d r \leq C \int_{0}^{1} s^{p \beta}(1-s)^{-1} \varphi^{p}(s) M_{q}^{p}\left(s, R_{\beta} f\right) d s .
$$

Set $h(r)=r^{-1} M_{q}(r, f)$. Since $R_{\beta}=R_{\beta, 0}$ and $1 \leq r^{-u}$ for any $r \in(0,1)$ and $u>0$, Proposition 3.1 implies

$$
\begin{aligned}
& \int_{0}^{1}(1-r)^{-1} \varphi^{p}(r) M_{q}^{p}\left(r, R_{\beta} f\right) d r \\
& \quad \leq C \int_{0}^{1} r^{p \beta}(1-r)^{-1} \varphi^{p}(r)\left\{\int_{0}^{1}\left(\log \frac{1}{\varrho}\right)^{\beta u-1} \varrho^{u-1} h(r \varrho)^{u} d \varrho\right\}^{p / u} d r .
\end{aligned}
$$

By Lemma 2.3 and Proposition 3.4, this can be controlled by

$$
C \int_{0}^{1} r^{-p}(1-r)^{p \beta-1} \varphi^{p}(r) M_{q}^{p}(r, f) d r .
$$

That is,

$$
\int_{0}^{1}(1-r)^{-1} \varphi^{p}(r) M_{q}^{p}\left(r, R_{\beta} f\right) d r \leq C \int_{0}^{1} r^{-p}(1-r)^{p \beta-1} \varphi^{p}(r) M_{q}^{p}(r, f) d r .
$$

Assume that $f(0)=0$; then $f=R_{\beta}\left(R^{\beta} f\right)$. By replacing $f$ with $R^{\beta} f$ in the above inequality, we get

$$
\begin{aligned}
\int_{0}^{1}(1-r)^{-1} \varphi^{p}(r) M_{q}^{p}(r, f) d r & \lesssim \int_{0}^{1}(1-r)^{-1} \varphi^{p}(r) M_{q}^{p}\left(r, R_{\beta}\left(R^{\beta} f\right)\right) d r \\
& \lesssim \int_{0}^{1}(1-r)^{p \beta-1} \varphi^{p}(r) M_{q}^{p}\left(r, R^{\beta} f\right) d r .
\end{aligned}
$$

Now, replacing $f$ by $f-f(0)$, since $R^{\beta}(f-f(0))=R^{\beta} f$, we get

$$
\int_{0}^{1}(1-r)^{-1} \varphi^{p}(r) M_{q}^{p}(r, f) d r \lesssim|f(0)|+\int_{0}^{1}(1-r)^{p \beta-1} \varphi^{p}(r) M_{q}^{p}\left(r, R^{\beta} f\right) d r .
$$

When $p=\infty$, we claim that for any $s, u>0$,

$$
\int_{0}^{1} \frac{(\log (1 / \varrho))^{\beta u-1} \varrho^{s u-1}}{(1-r \varrho)^{\beta u} \varphi^{u}(r \varrho)} d \varrho \leq C \frac{1}{\varphi^{u}(r)} .
$$


In fact, by (2.5) and the inequality in [4, p. 625],

$$
\begin{aligned}
& \int_{0}^{1} \frac{(\log (1 / \varrho))^{\beta u-1} \varrho^{s u-1}}{(1-r \varrho)^{\beta u} \varphi^{u}(r \varrho)} d \varrho \\
& \quad \leq C \frac{(1-r)^{a u}}{\varphi^{u}(r)} \int_{0}^{1} \frac{(\log (1 / \varrho))^{\beta u-1} \varrho^{s u-1}}{(1-r \varrho)^{\beta u+a u}} d \varrho \leq C \frac{1}{\varphi^{u}(r)} .
\end{aligned}
$$

If $M_{q}\left(r, R^{\beta} f\right)=O\left(1 /\left((1-r)^{\beta} \varphi(r)\right)\right)$ as $r \rightarrow 1^{-}$, then from (3.1) and $(3.4)$,

$$
M_{q}(r, f-f(0)) \leq C\left\{\int_{0}^{1}\left(\log \frac{1}{\varrho}\right)^{\beta u-1} \varrho^{-1} M_{q}^{u}\left(r \varrho, R^{\beta} f\right) d \varrho\right\}^{1 / u} \leq C \frac{1}{\varphi(r)} .
$$

The "only if" part follows from Proposition 3.2.

Proof of Theorem 1.2. This follows from the obvious modification of the proof of Theorem 1.1. The details are omitted.

4. The extension of Theorem A. With the same approach as in the last section, we now extend Theorem A to the weighted case and to more general fractional derivative operators.

We define

$$
\begin{aligned}
J^{\beta, t} f(z) & =\sum_{k, v} \frac{\Gamma(k+\beta+t)}{\Gamma(k+t)} a_{k, \nu} \varphi_{k, \nu}(z), \\
J_{\beta, t} f(z) & =\sum_{k, v} \frac{\Gamma(k+t)}{\Gamma(k+\beta+t)} a_{k, \nu} \varphi_{k, \nu}(z) .
\end{aligned}
$$

In particular $f^{[\beta]}=J^{\beta, 1} f, f_{[\beta]}=J_{\beta, 1} f$. Clearly

$$
J^{\beta, t} f(z)=\left(f^{[\beta+t-1]}\right)_{[t-1]}, \quad J_{\beta, t} f(z)=\left(f_{[\beta+t-1]}\right)^{[t]} .
$$

It is known [5] that $f^{[\beta]}$ and $f_{[\beta]}$ are holomorphic on $\Omega$, hence so are $J^{\beta, t} f(z)$, $J_{\beta, t} f(z)$.

Theorem 4.1. Assume $f \in H(\Omega), \varphi$ is normal, $0<p, q \leq \infty, \beta>0$, $t>0$. Then

$$
\int_{0}^{1}(1-r)^{-1} \varphi^{p}(r) M_{q}^{p}(r, f) d r \simeq \int_{0}^{1}(1-r)^{p \beta-1} \varphi^{p}(r) M_{q}^{p}\left(r, J^{\beta, t} f\right) d r .
$$

When $p=\infty$, the inequality is understood to be its limit case:

$$
M_{q}(r, f)=O\left(\varphi^{-1}(r)\right) \Leftrightarrow M_{q}\left(r, J^{\beta, s} f\right)=O\left((1-r)^{-\beta} \varphi^{-1}(r)\right)
$$

as $r \rightarrow 1^{-}$. 
In the special case $\varphi(r)=(1-r)^{k}(k>0)$ and $J^{\beta, t}=J^{[\beta]}$, Theorem 4.1 belongs to Stoll [8] and Shi [6].

Proof of Theorem 4.1. We first prove the special case $J^{\beta, t} f=f^{[\beta]}$. The estimate " $\gtrsim$ " is trivial from the inequality $M_{q}\left(r^{2}, f^{[\beta]}\right) \leq C(1-r)^{-\beta} M_{q}(r, f)$ (see $[6])$.

To prove the converse, we apply Theorem 1 of [6], which can be written as

$$
r^{\beta} M_{q}\left(r, f_{[\beta]}\right) \leq C\left\{\int_{0}^{r}(r-\varrho)^{u \beta-1} M_{q}^{u}(r \varrho, f) d \varrho\right\}^{1 / u},
$$

where $u=\min \{q, 1\}$. It is evident that

$$
\int_{0}^{1}(1-r)^{-1} \varphi^{p}(r) M_{q}^{p}\left(r, f_{[\beta]}\right) d r \leq C \int_{0}^{1} r^{\beta}(1-r)^{-1} \varphi^{p}(r) M_{q}^{p}\left(r, f_{[\beta]}\right) d r .
$$

If $0<p<\infty$, then by (4.2) and Lemma 2.2 we have

$$
\begin{aligned}
& \int_{0}^{1}(1-r)^{-1} \varphi^{p}(r) M_{q}^{p}\left(r, f_{[\beta]}\right) d r \\
& \quad \leq C \int_{0}^{1}(1-r)^{-1} \varphi^{p}(r)\left\{\int_{0}^{r}(r-\varrho)^{u \beta-1} M_{q}^{u}(r \varrho, f) d \varrho\right\}^{p / u} d r \\
& \leq C \int_{0}^{1}(1-r)^{p \beta-1} \varphi^{p}(r) M_{q}^{p}(r, f) d r .
\end{aligned}
$$

Replacing $f$ by $f^{[\beta]}$ we get

$$
\int_{0}^{1}(1-r)^{-1} \varphi^{p}(r) M_{q}^{p}(r, f) d r \leq C \int_{0}^{1}(1-r)^{p \beta-1} \varphi^{p}(r) M_{q}^{p}\left(r, f^{[\beta]}\right) d r .
$$

If $p=\infty$, we claim that for any $u>0$,

$$
r^{-\beta}\left\{\int_{0}^{r} \frac{(r-\varrho)^{\beta u-1}}{(1-\varrho)^{\beta u} \varphi^{u}(\varrho)} d \varrho\right\}^{1 / u} \leq C \frac{1}{\varphi(r)} .
$$

Indeed,

$$
\begin{aligned}
r^{-u \beta} \int_{0}^{r} \frac{(r-\varrho)^{\beta u-1}}{(1-\varrho)^{\beta u} \varphi^{u}(\varrho)} d r & =\int_{0}^{1} \frac{(1-s)^{\beta u-1}}{(1-r s)^{\beta u} \varphi^{u}(r s)} d s \\
& \leq C \frac{(1-r)^{a u}}{\varphi^{u}(r)} \int_{0}^{1} \frac{(1-s)^{\beta u-1}}{(1-r s)^{\beta u+a u}} d s \leq C \frac{1}{\varphi^{u}(r)} .
\end{aligned}
$$


It follows from the claim and (4.2) that $M_{q}\left(r, f^{[\beta]}\right)=O\left(1 /\left((1-r)^{\beta} \varphi(r)\right)\right)$ implies $M_{q}(r, f)=O(1 / \varphi(r))$.

Now we consider the general case. Notice that $J^{\alpha, t} f=\left(f^{[\alpha+t-1]}\right)_{[t-1]}$; applying the just proved results twice we have

$$
\begin{aligned}
\int_{0}^{1}(1-r)^{-1} \varphi^{p}(r) M_{q}^{p}\left(r, J^{\alpha, t} f\right) d r & \simeq \int_{0}^{1}(1-r)^{p(s-1)-1} \varphi^{p}(r) M_{q}^{p}\left(r, f^{[\alpha+t-1]}\right) d r \\
& \simeq \int_{0}^{1}(1-r)^{-1} \varphi^{p}(r) M_{q}^{p}(r, f) d r .
\end{aligned}
$$

This completes the proof.

\section{References}

[1] S. Bochner, Classes of holomorphic functions of several variables in circular domains, Proc. Nat. Acad. Sci. U.S.A. 46 (1960), 721-723.

[2] J. Burbea, Boundary behavior of holomorphic functions in the ball, Pacific J. Math. 127 (1987), 1-17.

[3] K. T. Hahn and J. Mitchell, $H^{p}$ spaces on bounded symmetric domains, Ann. Polon. Math. 28 (1973), 89-95.

[4] L. K. Hua, Harmonic Analysis of Functions of Several Complex Variables in the Classical Domains, Transl. Math. Monogr. 6, Amer. Math. Soc., 1963.

[5] J. Mitchell and K. T. Hahn, Representation of linear functionals in $H^{p}$ spaces over bounded symmetric domains in $C^{n}$, J. Math. Anal. Appl. 56 (1976), 379-396.

[6] J. H. Shi, On the rate of growth of the means $M_{p}$ of holomorphic and pluriharmonic functions on bounded symmetric domains of $C^{n}$, ibid. 126 (1987), 161-175.

[7] A. L. Shields and D. L. Williams, Bounded projections, duality and multipliers in spaces of analytic functions, Trans. Amer. Math. Soc. 167 (1971), 287-302.

[8] M. Stoll, On the rate of growth of the means $M_{p}$ of holomorphic and pluriharmonic functions on the ball, J. Math. Anal. Appl. 93 (1983), 109-127.

[9] K. H. Zhu, Multipliers of BMO in the Bergman metric with applications to Toeplitz operators, J. Funct. Anal. 87 (1989), 31-50.

Department of Mathematics

University of Science and Technology of China

Hefei, Anhui, 230026, P.R. China

E-mail: rengb@ustc.edu.cn
Department of Mathematics University of Aveiro 3810-193 Aveiro, Portugal

E-mail: uwek@mat.ua.pt

Received March 4, 2002

Revised version December 2, 2002 